\title{
Developmental Dynamics of Globodera ellingtonae in Field-Grown Potato
}

\author{
Wendy S. Phillips, Megan Kitner, and Inga A. Zasada, United States Department of Agriculture-Agricultural Research Service Horticultural \\ Crops Research Laboratory, Corvallis, OR 97330
}

\begin{abstract}
Globodera ellingtonae is a recently described nematode parasite of potato, which is closely related to the economically significant potato cyst nematodes G. rostochiensis and G. pallida. Because of the close relationship of $G$. ellingtonae to the potato cyst nematodes, a greater understanding of its biology is critical. Two experiments were conducted in Oregon to explore the developmental biology of G. ellingtonae in field-grown potato. The first experiment was conducted in 2013 and 2014 to determine the developmental timing of G. ellingtonae life stages and reproduction by inoculating potato with soil containing cysts followed by weekly collection of soil and root samples. Life stages; second-stage juveniles (J2) in soil and roots, third-stage juveniles (J3) and fourth-stage (J4) females and males in roots, males and females or cysts in soil, and egg number and developmental state were quantified. Normalizing across years using accumulated developmental degree days above $6^{\circ} \mathrm{C}$ (DD6), J2 of G. ellingtonae were found in soil from 41 to 588 DD6; two peaks of J2 invasion of roots were observed. The first adult females were observed at 387 and

449 DD6 in 2013 and 2014, respectively. The next generation of eggs was first observed from 675 to 854 DD6 and 50\% egg development (containing a vermiform juvenile) occurred at approximately 920 DD6. A second J2 hatch was observed in both years at 927 to 1,073 DD6. The developmental dynamics of $G$. ellingtonae observed here are similar to those reported for G. rostochiensis and G. pallida from several geographical locations. In the second experiment, the effect of potato and bare soil on G. ellingtonae egg hatch was evaluated; in 2014 and 2015, packages containing cysts in soil were buried under potato or in bare soil at the time of planting and eggs per cyst determined weekly. Across years, a significant reduction in eggs per cysts under potato $(>50 \%)$ was observed 35 days after planting (DAP) and, at 63 DAP, eggs per cyst were reduced by 76 to $96 \%$ compared with initial egg per cyst densities. In bare soil, the maximum reduction in densities of eggs per cyst was 55 to $73 \%$. This annual reduction in egg numbers of G. ellingtonae in bare soil is similar to that reported for G. pallida and G. rostochiensis.
\end{abstract}

The potato cyst nematodes Globodera pallida and G. rostochiensis are globally important pathogens of potato. Both nematodes are found on every continent (except Antarctica) and are quarantine pests in many countries (CABI 2015). In the United Kingdom, it has been estimated that G. pallida and G. rostochiensis cause a $9 \%$ loss of potato yield annually (Moxnes and Hausken 2007). In the United States, G. rostochiensis was first reported in New York in the 1940s (Chitwood et al. 1942), whereas G. pallida was more recently found in Idaho in 2006 (Hafez et al. 2007). As part of a nationwide survey of potato cyst nematodes in the United States triggered by the find of G. pallida in Idaho, populations of unusual Globodera nematodes were found in two fields in Idaho with histories of potato production. Independent of these finds, another population of unusual Globodera nematodes was found at a research facility in Oregon. These populations were subsequently used to describe a new species of potato cyst nematode, G. ellingtonae (Handoo et al. 2012). Morphological and phylogenetic evidence indicate that $G$. ellingtonae also occurs in Chile and Argentina (Lax et al. 2014; Skantar et al. 2011). Because this nematode is biologically and phylogenetically closely related to the quarantine nematodes G. pallida and G. rostochiensis (Skantar et al. 2011; Zasada et al. 2013, 2015) a greater understanding of the biology of G. ellingtonae is critical.

Potato cyst nematodes are sedentary endoparasites with narrow host ranges, and their global success can be attributed to several unique survival strategies. Similar to other plant-parasitic nematodes, potato cyst nematodes have an egg, four juvenile, and adult stages (male and female). Hatch of the second-stage juvenile (J2) from an egg is stimulated by host root diffusates (Perry 1989). The J2 then invades the root, migrates in the root cortex, and establishes a permanent feeding site in the pericycle, cortex, or endodermis (Turner and

Corresponding author: I. Zasada; E-mail: inga.zasada@ars.usda.gov

Accepted for publication 15 March 2017.

This article is in the public domain and not copyrightable. It may be freely reprinted with customary crediting of the source. The American Phytopathological Society, 2017.
Evans 1995). Development to third-stage (J3) and fourth-stage (J4) juveniles continues in the root, with sex differentiation occurring at the J3 stage. Males emerge from the root after the final molt while females remain in the root and increase in size. Fertilized females eventually erupt from the root while filling with developing eggs. The cuticle of the female forms a tough protective cyst, containing 200 to 500 eggs that can survive in soil for up to 30 years (Turner 1996).

There is general agreement that $G$. rostochiensis can develop at higher temperatures than G. pallida, and G. pallida at lower temperatures than G. rostochiensis (Foot 1978; Kaczmarek et al. 2014; Mugniery 1978). We have demonstrated in experiments under controlled environment conditions that $G$. ellingtonae has developmental dynamics similar to those of $G$. rostochiensis for life cycle completion (Phillips et al. 2015). Setting a base temperature of $6^{\circ} \mathrm{C}$, between 784 and 884 degree days were required for the completion of a complete life cycle (egg to egg). Understanding when key developmental events of G. ellingtonae occur in a field environment will allow for prediction of risk for this nematode to survive and complete its life cycle in other geographic locations and also will allow for the development of management practices aimed at disrupting the nematode's life cycle. For example, knowing when $\mathrm{J} 2$ hatch occurs and when $\mathrm{J} 2$ are present in soil may allow for the application of management strategies before the nematode enters the root, or targeted planting of trap crops to coincide with the nematode life cycle (Scholte and Vos 2000).

The goal of this research program is to provide biological data on G. ellingtonae upon which to make regulatory decisions and to develop management strategies against this new cyst nematode. In this study, we planted potato seed pieces with soil containing inoculum of G. ellingtonae and monitored nematode developmental stages in roots and soil to find key developmental time frames for management decisions. Additionally, to enable prediction of population dynamics of $G$. ellingtonae over a longer time frame, we tracked the reduction in egg numbers under potato and in bare soil.

\section{Materials and Methods}

Field environment and agronomic logistics. Field trials were carried out in 2013, 2014, and 2015 at the Oregon State University Central Oregon Agricultural Research Center, Powell Butte. The soil 
at Powell Butte, OR is a Redmond ashy sandy loam, and the climate is warm-summer Mediterranean, with average annual rainfall of $27 \mathrm{~cm}$. Before planting potato, the entire area was treated with the preemergence herbicide S-ethyl dipropylthiocarbamate (Eptam; Gowan Company, Yuma, AZ) at a rate of 4.1 liters/ha, and triple16 fertilizer (16-16-16, N-P-K) at 1,344 kg/ha prior to shallow cultivation. In 2013 and 2014 and 2015, 'Russet Burbank' and 'Russet Ranger' potato, respectively, were planted in furrows $86 \mathrm{~cm}$ apart in a section of field where $G$. ellingtonae was previously undetected. Cut potato seed pieces, approximately $56 \mathrm{~g}$, were treated with mancozeb (Maxim MZ; Syngenta Crop Protection, Greensboro, NC) to protect seed pieces from soilborne pathogens prior to being placed in furrows at approximately $25 \mathrm{~cm}$. Inoculation of seed pieces with G. ellingtonae varied with experiment and is described in detail below. After hand planting, the tubers were treated with the insecticide imidicloprid (Alias 4F; Adama, Aventura, FL) at 0.44 liter/ha and the fungicide azoxystroblin (Quadris; Syngenta Crop Protection) at 0.9 liter/ha applied in a $15-\mathrm{cm}$ band over the seed, and then the rows were covered with additional soil using a tractor-mounted disk hiller. Potato seed pieces were planted 20 May, 19 May, and 14 May in 2013, 2014 , and 2015, respectively. The trial area was overhead sprinkler irrigated starting approximately 4 to 5 weeks after planting, and potato was managed with cultural practices common in central Oregon. To control early blight (Alternaria solani), plants were treated with boscalid (Endura; BASF, Florham Park, NJ) at 0.33 liter/ha and azoxystroblin at 0.88 liter/ha based upon a growing-degree-day model used in the region (Gent and Schwartz 2003). Weeds were additionally managed postplant with pendimethalin (Prowl; BASF) at 2.34 liter/ha. During all trials, soil temperatures were recorded every 15 min with two WatchDog data loggers (Spectrum Technologies, Aurora, IL) buried at a depth of $15 \mathrm{~cm}$ in a row within the experimental trials.

G. ellingtonae development in potato. Nematode inoculum for the trials was produced the corresponding previous field season by inoculating individual Russet Burbank potato pieces with G. ellingtonae cysts containing eggs in 22-liter pots (Grip Lip 2800; Nursery Supplies Inc., McMinnville, OR) buried in the field. The following spring, pots were removed from the ground and soil was emptied onto tarps, dried under shelter, thoroughly mixed, and sifted to remove rocks and large debris. Triplicate soil samples from the inoculum soil were tested for cyst and egg densities. Cysts with an average of $259 \pm 27$ and $307 \pm 34$ eggs/cyst were used for inoculum in 2013 and 2014, respectively.

Experiments were conducted in 2013 and 2014 in an area approximately $30 \mathrm{~m}$ long containing 16 rows of potato. At planting, a 250$\mathrm{cm}^{3}$ scoop of field soil with cysts containing a total of approximately 2,000 to 3,000 eggs at an average of 10 cysts per $250 \mathrm{~cm}^{3}$ of soil was placed underneath each seed piece in the furrow. Sampling began 1 to 2 weeks after planting, with five plants collected each week from different rows within the 16-row area. Individual plants were removed from the ground with a shovel to enable collection of as much of the root system as possible while minimizing disturbance to the soil clinging to the roots. At the same time, soil was also collected from around the roots of each plant. Soil and roots were placed in a bag or bucket and returned to the laboratory the same day as collection and stored at $4^{\circ} \mathrm{C}$ until being processed the next day.

In the laboratory, the top portion of the plant was removed and discarded. Roots were carefully removed from collected soil so that disturbance to the surface of roots was minimized. To determine the number of adult males and $\mathrm{J} 2$ in bulk soil, a 250-g subsample was collected. Additionally, to account for nematodes in rhizosphere soil, the soil clinging to the roots was rinsed with water into a 2-liter plastic beaker. Both samples were then wet-sieve extracted by pouring the solutions over nested 250 - and $25-\mu \mathrm{m}$ sieves, with nematodes being collected on the $25-\mu \mathrm{m}$ sieve, followed by sucrose gradient extraction (Byrd et al. 1966). Following sucrose centrifugation, nematodes were again collected on a $25-\mu \mathrm{m}$ sieve and washed into a 50-ml tube to a volume of $10 \mathrm{ml}$, and duplicate 1-ml subsamples were counted using an inverted compound microscope (Leica, Buffalo Grove, IL). Once adult females were expected to have developed, the $250-\mu \mathrm{m}$ sieve fraction from the rhizosphere soil wash was saved and all females were collected and counted as described above. Eggs from females were enumerated by crushing females with a rubber stopper on a125- $\mu \mathrm{m}$ sieve nested over a $25-\mu \mathrm{m}$ sieve, washing the fraction collected on the $25-\mu \mathrm{m}$ sieve with water into a $50-\mathrm{ml}$ tube, and counting duplicate $1-\mathrm{ml}$ samples. At the same time as enumerating eggs, the developmental state of the eggs was recorded, with the first 100 eggs counted categorized as either having a distinct vermiform-shaped juvenile within the egg ("developed") or not.

Collected roots, or a subsample of roots when samples were large, were washed, patted dry, weighed, stained with acid fuchsin, and stored in a 33\% glycerol-33\% lactic acid solution (Byrd et al. 1983). To extract stained nematodes from roots, roots were cut into approximately 3-mm pieces and processed with a blender in approximately $200 \mathrm{ml}$ of water for $20 \mathrm{~s}$ on a high setting. The blended solution was poured over nested 250-, 125-, and 25- $\mu \mathrm{m}$ sieves and washed well with water, and the contents of the sieves were washed separately into $50-\mathrm{ml}$ tubes and adjusted to $10-$ to $20-\mathrm{ml}$ volumes. Nematode life stages (J2, J3, J4 female, J4 male, and female) were enumerated from duplicate 1-ml samples from the 25 - and $125-\mu \mathrm{m}$ sieve fractions using an inverted compound microscope and the entirety of the $250-\mu \mathrm{m}$ sieve fraction using a dissecting microscope.

In 2013, cysts in soil collected from under plants were also characterized each week to track reduction in eggs per cyst due to hatch and increase in eggs per cyst due to reproduction. A subsample of bulk soil was dried on aluminum trays in a greenhouse. Cysts were then extracted from $500 \mathrm{~g}$ of the dried soil using a United States Department of Agriculture cyst extractor (Ayoub 1980). Both the number of cysts and eggs per cyst were determined. To enumerate eggs, cysts were crushed with a rubber stopper on a $250-\mu \mathrm{m}$ sieve, with released eggs retained on a $25-\mu \mathrm{m}$ sieve. Eggs were washed with water into a $50-\mathrm{ml}$ tube to a volume of $20 \mathrm{ml}$ and eggs were counted in duplicate 1-ml subsamples using an inverted microscope.

G. ellingtonae egg hatch under potato and in bare soil. In a second set of experiments in 2014 and 2015, packets containing cysts were buried under potato and in bare ground to track reductions in egg numbers per cyst. To obtain soil with high cyst densities to place in packages, the floating fraction from dried soil was extracted by placing soil in a bucket, covering with water, and collecting float. This material was spread in a thin layer, allowed to dry for 2 to 3 days, and then mixed back into a portion of the microplot soil. Packets of this high-cyst soil were constructed by placing $35 \mathrm{~g}$ of the soil in a $10-\mathrm{cm}$ square of $250-\mu \mathrm{m}$ nylon mesh (McMaster-Carr, Santa Fe Springs, CA) folded into a triangle and heat sealed to close (Jores Technology, Sunrise, FL). In 2014, packets contained approximately 50 cysts, with a mean of $307 \pm 34$ eggs/cyst; and, in 2015, packets contained approximately 75 cysts, with a mean of $311 \pm 26$ eggs/ cyst $(n=5)$. Potato seed pieces were planted as described above, with cyst packets being placed directly under the seed pieces. A furrow in bare soil, $30 \mathrm{~m}$ long, was also created $260 \mathrm{~cm}$ from the potato row in which cyst packets were placed at a spacing similar to that in the potato row, and the furrow was closed by disk.

Five cyst packets were collected weekly, beginning 1 week after planting and extending for 10 weeks, from randomly selected locations in potato and bare rows. The packets were transported to the laboratory the same day as collection and stored at $4^{\circ} \mathrm{C}$ until being processed within 2 days. To extract cysts, the packets were cut open and all contents were placed in a 2-liter beaker and vigorously mixed with approximately 1 liter of water. The solution was allowed to settle for $15 \mathrm{~s}$ before being poured over nested 250 - and $25-\mu \mathrm{m}$ sieves, with sediment retained in the beaker. The sediment was suspended and the suspension was decanted two more times, each time taking care to flush out cysts clinging to the sides of the beaker. For collection of cysts, the $250-\mu \mathrm{m}$ sieve contents were further size differentiated for the collection of slightly larger cyst sizes, while removing excess duff by passing through a nested 1,000- over 354- $\mu \mathrm{m}$ sieve stack. The $354-\mu \mathrm{m}$ sieve contents were washed onto filter paper, dried, and stored at room temperature until further processing. Cysts from each sample were collected and eggs per cyst were enumerated as described above. 
Statistical analyses. Accumulated degree days above $6^{\circ} \mathrm{C}$ (DD6) were calculated using daily mean temperatures. Eggs per cyst data from cysts collected underneath potato in 2013 and from packages under potato and in bare ground in 2014 and 2015 were square root transformed prior to analysis to correct for heteroscedasticity. The data were then analyzed for effects of time, treatment (potato versus bare), time-treatment interaction, and replication using a mixed linear model analysis of variance, with time and treatment as fixed factors and replication as a random factor. Treatment means within and across time were separated using Tukey's test $(P=0.05)$. All analyses were performed using JMP 9.1 (SAS Institute, Cary, NC).

\section{Results}

Yearly climate conditions. A large shift in mean soil temperature occurred early in the 2013 growing season, with temperatures increasing from $10^{\circ} \mathrm{C}$ at planting to $20^{\circ} \mathrm{C} 2$ weeks later (Fig. 1). Soil temperatures were more constant during the first 14 days after planting (DAP) in 2014 and 2015, ranging from 14.7 to 18.6 and 12.7 to $19.8^{\circ} \mathrm{C}$, respectively. Maximum soil temperatures recorded in 2013, 2014 , and 2015 were $27.0,23.5$, and $25.0^{\circ} \mathrm{C}$, respectively.

G. ellingtonae development in potato. The timing of $\mathrm{J} 2$ hatch varied between years. In 2013, the highest density of J2 in soil was recorded at the first sampling day, 14 DAP (129 DD6), and J2 were present in soil until 42 DAP (491 DD6) (Fig. 2A). In 2014, J2 appeared in soil at 21 DAP (239 DD6), with peak presence of J2 in soil at 35 DAP (381 DD6) and J2 remaining in soil until 49 DAP (533 DD6) (Fig. 2A). As another measure of hatching dynamics, the numbers of eggs in cysts were tracked in 2013 (Table 1). There was a significant decrease $(P<0.001)$ in eggs per cyst between 28 DAP (321 DD6) and 35 DAP (387 DD6), which corresponded with an increase in $\mathrm{J} 2$ in roots at 35 DAP (Fig. 3A).

The dynamics of the invasion of roots by $\mathrm{J} 2$ generally followed those of $\mathrm{J} 2$ in soil, with peak invasions occurring the week of or the week following peaks of $\mathrm{J} 2$ in soil. There were two peaks of initial J2 invasion of roots in 2013, the first peak at 14 DAP (129 DD6) and the higher, second peak at 35 DAP (387 DD6) (Fig. 3A). In 2014, there was an initial invasion of roots at approximately 21 DAP (239 DD6) to 28 DAP (301 DD6) but a much higher peak J2 population in roots occurred at 42 DAP (533 DD6) (Fig. 3B). In both years, advancement to the next juvenile stage appeared to occur at weekly intervals (Fig. 3A and B). In 2013, substantial root populations of J2 were already present at 14 DAP (129 DD6), J3 at 21 DAP (234 DD6), and J4 at 28 DAP (321 DD6), and males appeared in soil at 35 DAP (387 DD6) (Figs. 2B and 3A). Similarly, a second series of developmental stages appeared at weekly intervals following the second $\mathrm{J} 2$ root invasion in 2013 at 35 DAP (387 DAP), with peaks of J3 at 42 DAP (491 DD6) and J4 at 49 to 56 DAP (588 to 675 DD6), and a second peak of males in soil at 63 DAP (762 DD6). There was a similar timing for the progression of developmental stages in 2014 (Figs. 2B and 3B).

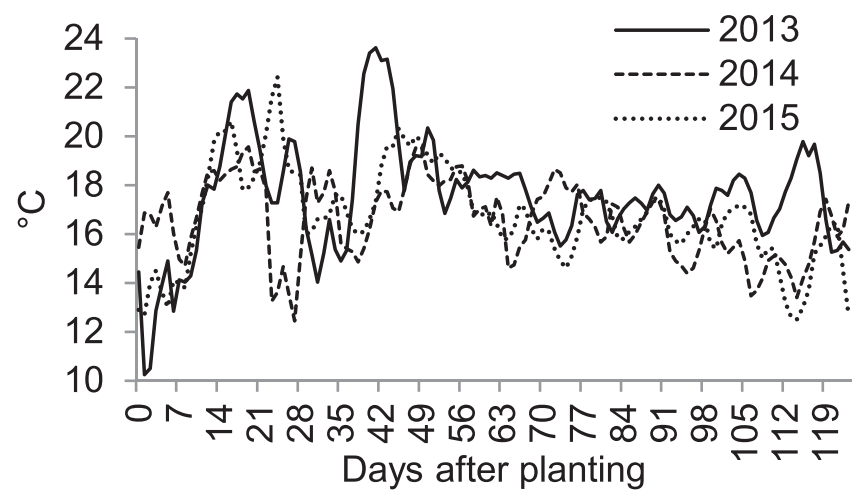

Fig. 1. Average daily soil temperature from planting to harvest of potato (Solanum tuberosum) for 3 years at Powell Butte, OR. Soil temperatures were measured at a depth of $15 \mathrm{~cm}$. Data points for each day are the average of two data loggers, each recording temperatures at 15 -min intervals.
The first adult females were observed at 36 DAP (387 DD6) in 2013 and 42 DAP (449 DD6) in 2014 (data not shown). Similar to the development of other life stages, eggs in females were seen earlier in 2013, at 56 DAP (675 DD6), than in 2014, when eggs were not recorded until 77 DAP (854 DD6) (Fig. 4A). The number of eggs per female at the end of the season in 2013 was much higher than in 2014 (Fig. 4A). By 77 and 84 DAP (916 and 927 DD6) in 2013 and 2014, respectively, over $50 \%$ of eggs contained vermiform juveniles (developed eggs) (Fig. 4B). At 98 DAP (1,147 and 1,065 DD6 in 2013 and 2014, respectively), over $80 \%$ of eggs were developed in both years, despite the number of eggs per female nearly doubling between 98 and 112 DAP (1,147 and 1,306 DD6) in 2013 (Fig. 4A). In 2013, there was a late-season hatch of J2, beginning at 91 DAP (1,073 DD6) (Fig. 2A). A late-season hatch also was observed in 2014 starting at 84 DAP (927 DD6).

G. ellingtonae egg hatch under potato and in bare soil. Across years, in general, a significant $(P<0.001)$ reduction $(>50 \%)$ in number of eggs per cyst occurred at 35 DAP (Table 1) under potato. In 2015 , the number of eggs per cyst under potato initially dropped at 21 DAP (218 DD6) compared with 14 DAP (125 DD6) but the larger reduction $(61 \%)$ compared with initial eggs per cyst occurred at 35 DAP (392 DD6). In 2013, number of eggs per cyst was monitored for a longer period of time under potato compared with 2014 and 2015. In 2013, after the initial reduction in eggs per cysts through 63 DAP (761 DD6), new eggs (next generation) were observed starting at 70 DAP (843 DD6). In 2014 and 2015, when number of eggs per cyst under potato versus in bare soil was considered, numbers of eggs per cysts were similar under these treatments through 28 DAP, after which there were significantly fewer eggs per cyst found under potato compared with in bare soil $(P<0.001$; Table 1$)$. There was a general decline in eggs per cyst in bare soil over the timeframe of the experiment (70 DAP), with an approximately $50 \%$ reduction in eggs per cyst during both years in bare soil.

\section{Discussion}

This is the first detailed description of the life cycle of G. ellingtonae in a field environment. $\mathrm{J} 2$ hatched from eggs at between 7 and 49 days, corresponding to 41 and 588 DD6, after planting of potato, with the development to subsequent life stages ( J3, J4, and adults) occurring at roughly weekly increments after invasion of roots by $\mathrm{J} 2$.



Fig. 2. Occurrence of Globodera ellingtonae $\mathbf{A}$, second-stage juveniles and $\mathbf{B}$, males in soil under potato (Solanum tuberosum) over time during two growing seasons at Powell Butte, OR. Each data point is the mean of five replicates. Error bars designate the standard errors of means. 
The time to at least $50 \%$ development of eggs occurred after the same amount of time in both years (916 and 927 DD6). Also in both years, a second flush of $\mathrm{J} 2$ in soil was observed late in the growing season, presumably from new eggs. Although it is difficult to make direct comparisons of G. ellingtonae development to the data on G. rostochiensis and G. pallida in the literature because of difference in biotic and abiotic conditions at experimental sites, some generalizations can be made. A summary of the developmental dynamics of Globodera spp. reported in the literature is presented in Table 2. Only studies where development was tracked in a field environment and samples were collected weekly were considered (Alonso et al. 2011; Bačić et al. 2011; Ebrahimi et al. 2014; Greco et al. 1988; Jiménez-Pérez et al. 2009; Mimee et al. 2015; Renčo 2007). This table will be referenced throughout the discussion to aid in comparing and contrasting G. ellingtonae development to that of G. pallida and G. rostochiensis.

Hatching factors, compounds produced by roots in the rhizosphere, are required to initiate hatch of Globodera spp. (Perry

Table 1. Change in densities of Globodera ellingtonae eggs per cyst over time under potato and in bare ground during 3 years at Powell Butte, OR

\begin{tabular}{|c|c|c|c|c|c|c|c|c|c|c|}
\hline \multirow[b]{3}{*}{$\mathbf{D A P} \mathbf{z}$} & \multirow{2}{*}{\multicolumn{2}{|c|}{$\begin{array}{c}2013 \\
\text { Potato }\end{array}$}} & \multicolumn{4}{|c|}{2014} & \multicolumn{4}{|c|}{2015} \\
\hline & & & \multicolumn{2}{|l|}{ Potato } & \multicolumn{2}{|c|}{ Bare ground } & \multicolumn{2}{|l|}{ Potato } & \multicolumn{2}{|c|}{ Bare ground } \\
\hline & Eggs/cyst & $\overline{\%}$ & Eggs/cyst & $\%$ & Eggs/cyst & $\%$ & Eggs/cyst & $\overline{\%}$ & Eggs/cyst & $\%$ \\
\hline 7 & $\ldots$ & $\ldots$ & $311( \pm 40) \mathrm{a}$ & 0 & $269( \pm 23) \mathrm{ab}$ & 0 & $353( \pm 20) \mathrm{ab}$ & 0 & $320( \pm 42) a-c$ & $\overline{0}$ \\
\hline 14 & $189( \pm 8)$ a & 26 & $305( \pm 29)$ a & 0 & $278( \pm 22)$ a & 0 & $367( \pm 23)$ a & 0 & $286( \pm 15) a-d$ & 8 \\
\hline 21 & $231( \pm 43)$ a & 11 & $202( \pm 16) a-c$ & 30 & $211( \pm 23) a b$ & 18 & $247( \pm 12) b-f$ & 20 & $298( \pm 16)$ a-d & 4 \\
\hline 28 & $205( \pm 17) \mathrm{a}$ & 21 & $242( \pm 38)$ ab & 8 & $198( \pm 20)$ abc & 28 & $258( \pm 18)$ b-e & 17 & $318( \pm 32)$ a $-d$ & 0 \\
\hline 35 & $64( \pm 12) b c$ & 75 & $112( \pm 26) \mathrm{c}-\mathrm{e}$ & 52 & $211( \pm 16) a b$ & 34 & $122( \pm 11) \mathrm{h}-\mathrm{k}$ & 61 & $237( \pm 15) \mathrm{c}-\mathrm{g}$ & 24 \\
\hline 42 & $46( \pm 6) b c$ & 82 & $39( \pm 7)$ ef & 85 & $152( \pm 22) b-d$ & 44 & $82( \pm 3) \mathrm{i}-1$ & 74 & $235( \pm 30) c-g$ & 24 \\
\hline 49 & $57( \pm 3) b c$ & 77 & $27( \pm 4) \mathrm{f}$ & 89 & $160( \pm 17) b-d$ & 53 & $85( \pm 9) \mathrm{i}-1$ & 73 & $211( \pm 6) d-h$ & 32 \\
\hline 56 & $24( \pm 8) c$ & 90 & $16( \pm 2) \mathrm{f}$ & 94 & $93( \pm 9) \mathrm{de}$ & 73 & $39( \pm 3) \mathrm{j}-1$ & 87 & $169( \pm 12) \mathrm{e}-\mathrm{i}$ & 46 \\
\hline 63 & $61( \pm 19) b c$ & 76 & $13( \pm 2) \mathrm{f}$ & 95 & $111( \pm 21) \mathrm{c}-\mathrm{e}$ & 63 & $11( \pm 3) 1$ & 96 & $139( \pm 35) \mathrm{g}-\mathrm{j}$ & 55 \\
\hline 70 & $108( \pm 14) a b$ & 58 & $28( \pm 3) \mathrm{f}$ & 89 & $147( \pm 11) b-d$ & 55 & $23( \pm 12) \mathrm{kl}$ & 92 & $145( \pm 20) \mathrm{f}-\mathrm{j}$ & 53 \\
\hline 77 & $203( \pm 27)$ a & 21 & $\ldots$ & $\ldots$ & $\ldots$ & $\ldots$ & $\ldots$ & $\ldots$ & $\ldots$ & $\ldots$ \\
\hline 84 & $197( \pm 21)$ a & 23 & $\ldots$ & $\ldots$ & $\ldots$ & $\ldots$ & $\ldots$ & $\ldots$ & $\ldots$ & $\ldots$ \\
\hline 91 & $209( \pm 19) \mathrm{a}$ & 19 & $\ldots$ & $\ldots$ & $\ldots$ & $\ldots$ & $\ldots$ & $\ldots$ & $\ldots$ & $\ldots$ \\
\hline
\end{tabular}

${ }^{y}$ Values are means of five (2013 and 2014) and four (2015) replicates followed by standard errors. Values within a column followed by the same letter are not significantly different according to Tukey's adjustment for multiple comparisons $(P \geq 0.05)$. Percent $(\%)$ indicates percentage reduction in eggs per cysts from original number of eggs per cyst of $259( \pm 27), 307( \pm 34)$, and $311( \pm 26)$ in 2013, 2014 and 2015, respectively $(n=5)$.

${ }^{z}$ Days after planting.

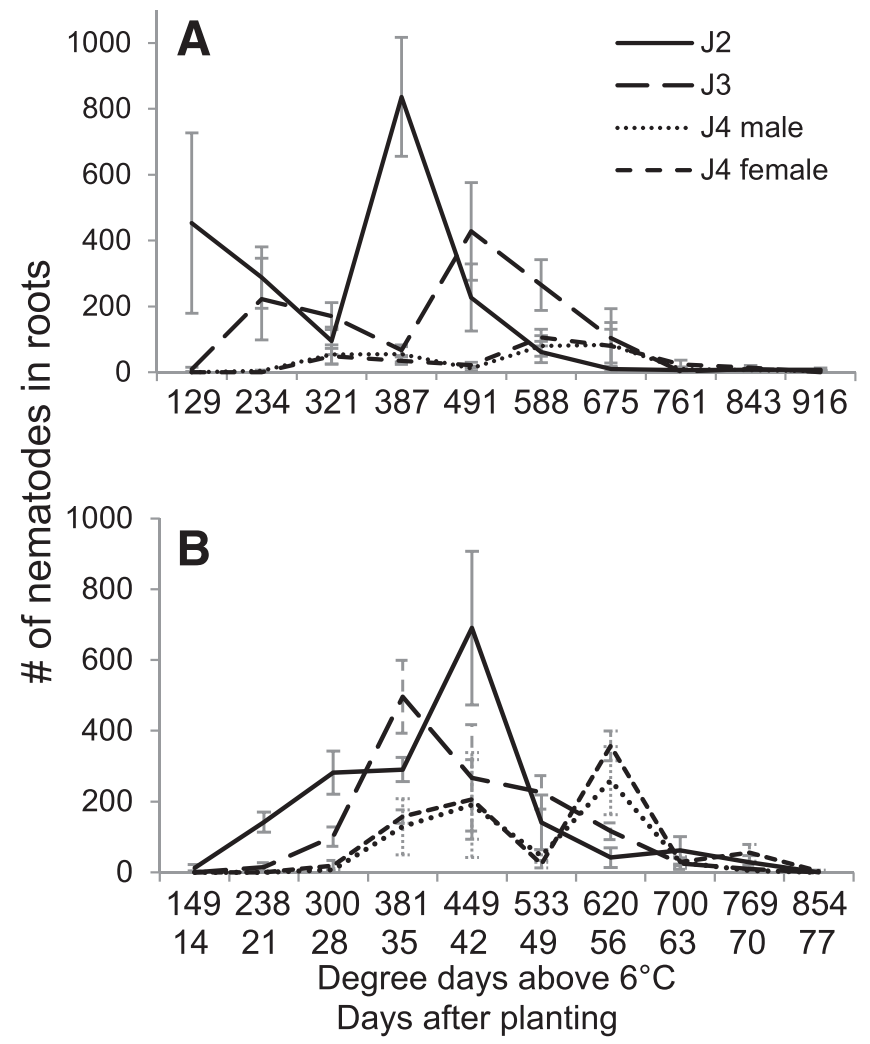

Fig. 3. Population dynamics of Globodera ellingtonae second-stage (J2) and thirdstage (J3) juveniles and fourth-stage (J4) juvenile males and females in roots of potato (Solanum tuberosum) in A, 2013 and B, 2014 at Powell Butte, OR. Each data point is the mean number of each stage in the total root system of one plant from five replicates. Error bars designate the standard errors of means.
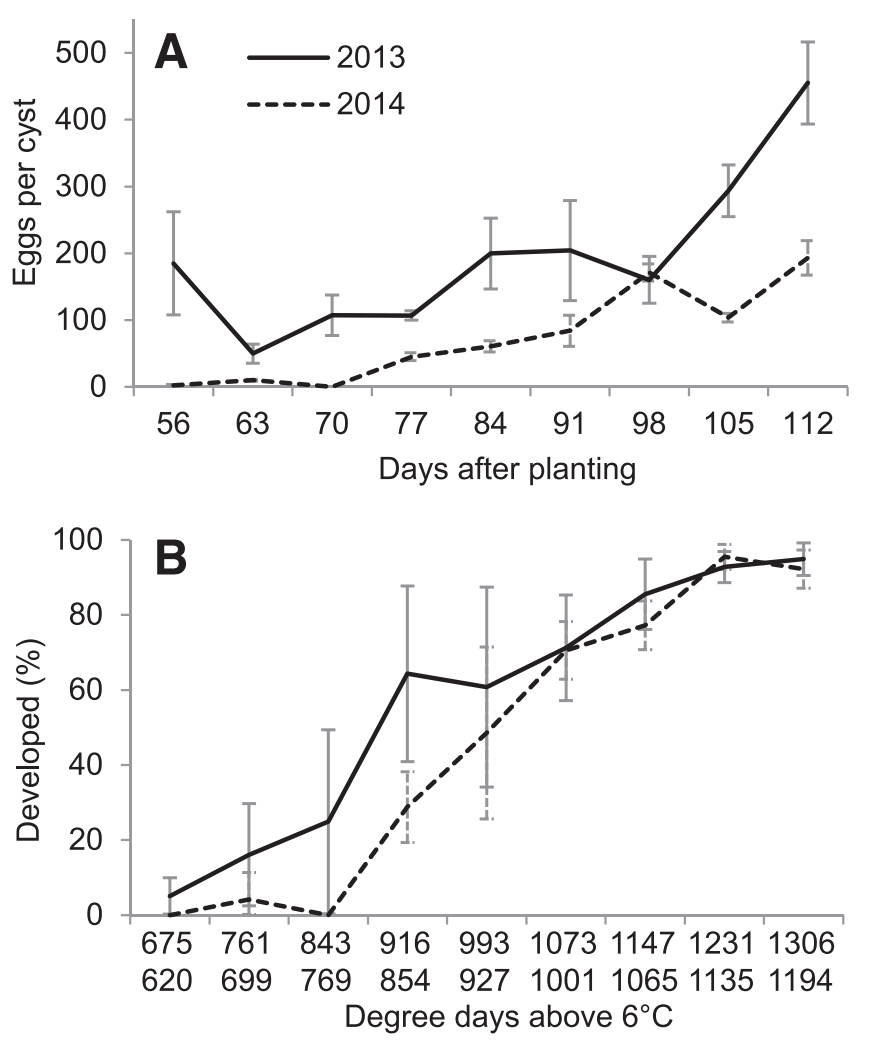

Fig. 4. A, Density of eggs per cyst in Globodera ellingtonae females associated with the roots of potato (Solanum tuberosum), and B, proportion of developed eggs (containing vermiform juveniles) in adult $\mathrm{G}$. ellingtonae females. Both variables were measured over time in 2013 and 2014 at Powell Butte, OR. Each data point is the mean of five replicates. Error bars designate the standard errors of means. 
1989). We previously demonstrated in laboratory assays that $G$. ellingtonae hatches vary rapidly after exposure to potato root diffusate, with $66 \%$ of eggs hatching within 3 days of exposure (Zasada et al. 2013). In addition to hatching factors, some have proposed that a specific number of degree days must accumulate before hatch and invasion (Jones and Perry 1978). Soil temperatures at and after planting $\left(10\right.$ to $\left.22^{\circ} \mathrm{C}\right)$ were well within the range where $G$. ellingtonae hatch would be expected to occur (Phillips et al. 2015). In another study (Renčo 2007), the presence of J2 of G. rostochiensis in soil ranged from 21 to 28 DAP (Table 2). At two locations in Canada, G. rostochiensis $\mathrm{J} 2$ were found in soil 14 to 28 DAP (Mimee et al. 2015).

Once hatched, G. ellingtonae $\mathrm{J} 2$ invaded roots quickly, similar to what has been observed for G. rostochiensis, and for G. pallida in Belgium (Table 2). In 2013, samples were not collected until 14 DAP, and the initial invasion of roots by $\mathrm{J} 2$ was potentially not captured. In 2014, we began sampling at 7 DAP but J2 were not observed in roots until 21 DAP. The number of DAP reported for $G$. rostochiensis $\mathrm{J} 2$ to be observed in roots after planting ranged from 7 to 35 days (Bačić et al. 2011; Ebrahimi et al. 2014; Greco et al. 1988; Jiménez-Pérez et al. 2009; Mimee et al. 2015; Renčo 2007). For G. pallida, Alonso et al. (2011) reported invasion occurring 56 to 57 DAP; however, plants did not emerge until that same time. In our study, it appeared that there were two waves of initial $\mathrm{J} 2$ invasion roughly separated by 14 to 21 days. This has not been widely reported in other studies examining Globodera developmental dynamics. Our combined growth chamber (Phillips et al. 2015) and field data indicate that multiple punctuated hatches within a growing season from the same generation of cysts may be common in G. ellingtonae.

We previously performed growth chamber studies comparing $G$. ellingtonae development at constant temperatures of 10 to $26.5^{\circ} \mathrm{C}$, where days to developmental stages varied greatly between temperatures but DD6 at which developmental stages were observed remained fairly consistent (Phillips et al. 2015). The DD6 to first presence of developmental stages were very similar between the growth chamber studies and the field in 2013: J2 in roots were initially seen approximately 100 DD6, J3 at 200 to 230 DD6, and males in soil at approximately 400 DD6. Due to delayed hatch, the DD6 from planting to developmental stages were later in 2014 but the DD6 between stages was similar to that observed in 2013. Development of the next generation of eggs occurred slightly earlier under growth chamber conditions, with approximately $10 \%$ developed eggs at approximately 650 DD6 and 50\% developed eggs at 784 to 884 DD6, as compared with approximately 700 and 900 DD6, respectively, for both years in the field. In studies with $G$. rostochiensis and $G$. pallida, reports of the first sign of eggs range from 42 to 68 DAP (Ebrahimi et al. 2014; Greco et al. 1988; Jiménez-Pérez et al. 2009; Mimee et al. 2015; Renčo 2007), similar to what we report here for G. ellingtonae and also what is reported for G. pallida in Belgium (Ebrahimi et al. 2014).

Unfortunately, the base temperatures used for accumulated degree day calculations have been inconsistent between Globodera studies. For example, in studies considering the development of G. rostochiensis, base temperatures of 5.9 to $6^{\circ} \mathrm{C}$ (Ebrahimi et al. 2014; Mimee et al. 2015) and $10^{\circ} \mathrm{C}$ (Greco et al. 1988; Greco and Moreno 1992) have been used. Based upon our growth chamber experiments (Phillips et al. 2015), $6^{\circ} \mathrm{C}$ was chosen as the base developmental temperature for G. ellingtonae. As noted in our growth chamber study, the developmental progress we observed for G. ellingtonae in roots was very similar to that observed for G. rostochiensis and G. pallida in Belgium (Ebrahimi et al. 2014). The DD6 to first appearance of each developmental stage in the 2013 field trial also was similar to that observed in Belgium for G. rostochiensis.

During both years, we observed a second flush of G. ellingtonae J2 in soil beginning at approximately 77 to 84 DAP. This hatching event was of greater magnitude in 2013 compared with 2014. Others have reported a second $\mathbf{J} 2$ hatch within a growing season. There was a second $\mathrm{J} 2$ hatch and invasion of roots of G. rostochiensis at 91 to 112 DAP in Slovakia (Renčo 2007), at approximately 95 DAP in Italy (Greco et al. 1988), and at 75 to 100 DAP in Canada (Mimee et al. 2015). In Central Chile, where potato crops can be cultivated for most of the year, a second $\mathrm{J} 2$ hatch of $G$. rostochiensis only occurred in spring-planted potato, not in summer- and winter-planted potato (Greco and Moreno 1992). Similar to our finding that there was no evidence of high levels of successful invasion of roots or subsequent development from the secondary $\mathrm{J} 2$ hatch in Oregon, Greco and Moreno (1992) also reported a lack of development of G. rostochiensis $\mathrm{J} 2$ at the end of the spring potato season in Chile, suggesting a single generation in both of these environments. However, in southern Italy and in Venezuela, G. rostochiensis has been reported to be able to complete a second generation within a growing season (Greco et al. 1988; Jiménez-Pérez et al. 2009). Globodera spp. are native to South America (Grenier et al. 2010), and this second J2 hatch may be a relic of origins in warmer South American environments where more than one generation per year is possible. The risk associated with a second $\mathrm{J} 2$ hatch in warmer environments, even when potato is not grown back to back, is the potential for solanaceous weeds that are hosts for Globodera spp. (Boydston et al. 2010; Mimee et al. 2015) to allow for the continued development of the nematode beyond a single generation. For G. ellingtonae, an alternative host that has the potential to support invasion of a second generation is hairy nightshade (W. S. Phillips and I. A. Zasada, unpublished data).

An understanding of the natural decline in Globodera eggs is an important component of developing rotational schemes in the absence of a host. We observed an approximately 50\% reduction in number of $G$. ellingtonae eggs per cyst in bare soil over the course

Table 2. Comparison of the developmental dynamics of Globodera ellingtonae at Powell Butte, OR with that of G. rostochiensis and G. pallida at various locations reported in the literature ${ }^{\mathrm{x}}$

\begin{tabular}{|c|c|c|c|c|c|c|c|c|c|}
\hline \multirow[b]{3}{*}{ Stage $^{\mathrm{z}}$} & \multirow{3}{*}{$\begin{array}{c}\text { G. ellingtonae } \\
\text { Oregon }\end{array}$} & \multicolumn{6}{|c|}{ Days after planting (degree days) ${ }^{\mathbf{y}}$} & & \\
\hline & & & & G. rostoc & & & & \multicolumn{2}{|c|}{ G. pallida } \\
\hline & & Serbia & Slov & Canada & Ven & Italy & $\overline{\text { Belgium }}$ & Belgium & Spain \\
\hline J2 soil & $7-21(129-239)$ & 7 & $14-21$ & $14-28(122)$ & 14 & 16 & 24 & $21(87)$ & $29-35(211-234)$ \\
\hline $\mathrm{J} 2$ root & $14-21(129-239)$ & 7 & 27 & $\ldots$ & 14 & 16 & 35 & 24 & $56-57(348-411)$ \\
\hline $\mathrm{J} 3$ & $21-28(234-300)$ & $21-28$ & 34 & $\ldots$ & 21 & 26 & 35 & 35 & $\ldots$ \\
\hline $\mathrm{J} 4$ & $28-35(321-381)$ & 28 & 40 & $\ldots$ & 28 & 34 & 49 & 49 & $\ldots$ \\
\hline Male soil & 28-42 (387-449) & 28 & 46 & $35-42(299)$ & 35 & 46 & $59(391)$ & $59(391)$ & $77(541-548)$ \\
\hline Female & $36-42(387-449)$ & 35 & 43 & 28-42 (299) & 35 & 46 & 59 (391) & $59(391)$ & 77 (541-548) \\
\hline Egg & $56-77(675-854)$ & $\ldots$ & 55 & $42-63(579)$ & 42 & 56 & $68(401)$ & $65(463)$ & $\ldots$ \\
\hline
\end{tabular}

× Literature considered from left to right: G. rostochiensis in Serbia (Bačić et al. 2011), Slovakia (Slov) (Renčo 2007), Canada (Mimee et al. 2015), Venezuela (Ven) (Jiménez-Pérez et al. 2009), Italy (Greco et al. 1988), and Belgium (Ebrahimi et al. 2014); and G. pallida in Belgium (Ebrahimi et al. 2014) and Spain (Alonso et al. 2011).

${ }^{y}$ Degree days presented when available. Degree days for G. ellingtonae calculated using a base temperature of $6^{\circ} \mathrm{C}$ (Phillips et al. 2015), for G. rostochiensis calculated using a base temperature of $5.9^{\circ} \mathrm{C}$, and for G. pallida calculated using a base temperature of $3.9^{\circ} \mathrm{C}$. Values presented are the days after planting or degree days of first occurrence of a life stage. If a range is presented, then data from multiple years were included.

${ }^{\mathrm{z}}$ Globodera life stages: $\mathrm{J} 2=$ second stage juvenile, $\mathrm{J} 3=$ third-stage juvenile, and $\mathrm{J} 4=$ fourth-stage juvenile. 
of 10 weeks. This reduction is similar to that which has been reported for G. pallida and G. rostochiensis. The annual rate of egg decline varied with soil type in New Zealand, with annual attrition rates of 30 to $70 \%$ for G. pallida and 45 to $61 \%$ for G. rostochiensis (Marshall 1998). In another study comparing G. pallida and G. rostochiensis hatch in the absence of a host (Ryan and Devine 2005), initially there was a difference in the rate of spontaneous hatch between the two species, with G. rostochiensis hatching in greater numbers than G. pallida; however, by the end of the study, the reduction in egg numbers was similar between the two species (37\%). In a 2 -year study, the decline in egg numbers of $G$. rostochiensis was $57 \%$ in the first year and $40 \%$ in the second year in the absence of a host (Devine et al. 1999). In this same study, it was discovered that hatch accounted for approximately $75 \%$ of this decline and egg mortality for an additional 10\%. Many factors, acting alone or together, can influence the decline in Globodera egg numbers in a field environment, including temperature and egg-degrading microorgnisms (Devine et al. 1999). The amount of time required for the complete hatch or death of G. ellingtonae eggs in the absence of a host is unknown.

Our data provide key insights into the developmental biology of G. ellingtonae. From a practical perspective, these data provide evidence for potential avenues to pursue from both management and regulatory perspectives. For example, our field data plus our previous observations on egg hatch of G. ellingtonae (Phillips et al. 2015; Zasada et al. 2013) indicate that $G$. ellingtonae $\mathbf{J} 2$ hatch and invade roots rapidly under suitable conditions. Understanding the time frame at which $\mathrm{J} 2$ are expected in soil will allow for the targeted application of nematicides. Our data also indicate that approximately 600 DD6 after planting is an optimal time to terminate a trap crop to achieve greatest reduction of old eggs before new eggs develop.

\section{Acknowledgments}

We thank the Northwest Potato Consortium, United States Department of Agriculture (USDA)-Agricultural Research Service, and USDA-Animal and Plant Health Inspection Service for funding this research; and A. Peetz, A. Howland, M. Ballato, N. Peterson, S. Kieran, B. Hartnell, and W. Sato for contributing to this research endeavor.

\section{Literature Cited}

Alonso, R., Alemany, A., and Andres, M. F. 2011. Population dynamics of Globodera pallida (Nematoda: Heteroderidae) on two potato cultivars in natural field conditions in Balearic Islands, Spain. Span. J. Agric. Res. 9:589-592.

Ayoub, S. M. 1980. Plant Nematology, An Agricultural Training Aid. NemaAid Publications, Sacramento, CA.

Bačić, J., Barsi, L., and Štrbac, P. 2011. Life cycle of the potato cyst nematode (Globodera rostochiensis) grown under climatic conditions in Belgrade. Arch. Biol. Sci. (Belgrade) 63:1069-1075.

Boydston, R. A., Mojtahedi, H., Bates, C., Zemetra, R., and Brown, C. R. 2010. Weed hosts of Globodera pallida in Idaho. Plant Dis. 94:918.

Byrd, D. W., Jr., Kirkpatrick, T., and Barker, K. R. 1983. An improved technique for clearing and staining plant tissue for detection of nematodes. J. Nematol. 14:142-143.

Byrd, D. W., Jr., Nusbaum, C. J., and Barker, K. R. 1966. A rapid flotation-sieving technique for extracting nematodes from soil. Plant Dis. Rep. 50:954-957.

CABI. 2015. Invasive Species Compendium. CAB International, Wallingford, UK.

Chitwood, B. G., Clement, R. L., Morgan, R., and Tank, R. 1942. Heterodera rostochiensis, golden nematode of potatoes in New York state. U.S. Dep. Agric. Plant Dis. Rep. 26:390-391.

Devine, K. J., Dunne, C., O'Gara, F., and Jones, P. W. 1999. The influence of inegg mortality and spontaneous hatching on the decline of Globodera rostochiensis during crop rotation in the absence of the host potato crop in the field. Nematology 1:637-645.

Ebrahimi, N., Viaene, N., Demeulemeester, K., and Moens, M. 2014. Observations on the life cycle of potato cyst nematodes, Globodera rostochiensis and $G$. pallida, on early potato cultivars. Nematology 16:937-952.
Foot, M. A. 1978. Temperature responses of three potato-cyst nematode populations from New Zealand. Nematologica 24:412-417.

Gent, D. H., and Schwartz, H. F. 2003. Validation of potato early blight disease forecast models for Colorado using various sources of meteorological data. Plant Dis. 87:78-84.

Greco, N., Inserra, R. N., Brandonisio, A., Tirro, A., and De Marinis, G. 1988 Life-cycle of Globodera rostochiensis on potato in Italy. Nematol. Mediterr. 16:69-73.

Greco, N., and Moreno, L. 1992. Development of Globodera rostochiensis during three different growing seasons in Chile. Nematropica 22:175-181.

Grenier, E., Fournet, S., Petit, E., and Anthoine, G. 2010. A cyst nematode 'species factory' called the Andes. Nematology 12:163-169.

Hafez, S. L., Sundararaj, P., Handoo, Z. A., Skantar, A. M., Carta, L. K., and Chitwood, D. J. 2007. First report of the pale cyst nematode, Globodera pallida, in the United States. Plant Dis. 91:325.

Handoo, Z. A., Carta, L. K., Skantar, A. M., and Chitwood, D. J. 2012. Description of Globodera ellingtonae n. sp. (Nematoda: Heteroderidae) from Oregon. J. Nematol. 44:40-57.

Jiménez-Pérez, N., Crozzoli, R., and Greco, N. 2009. The biology of Globodera rostochiensis in cultivated potato in Venezuela. Nematol. Mediterr. 37: 155-160.

Jones, F. G. W., and Perry, J. M. 1978. Modeling populations of cyst nematodes (Nematoda: Heteroderidae). J. Appl. Ecol. 15:349-371.

Kaczmarek, A., MacKenzie, K., Kettle, H., and Blok, V. C. 2014. Influence of soil temperature on Globodera rostochiensis and Globodera pallida. Phytopathol. Mediterr. 53:396-405

Lax, P., Rondan Dueñas, J. C., Franco-Ponce, J., Gardenai, C. N., and Doucet, M. E. 2014. Morphology and DNA sequence data reveal the presence of Globodera ellingtonae in the Andean region. Contrib. Zool. 83:227-243.

Marshall, J. W. 1998. Potato cyst nematodes (Globodera species) in New Zealand and Australia. Pages 353-394 in: Potato Cyst Nematodes: Biology, Distribution and Control. R. J. Marks and B. B. Brodie, eds. CABI International, Wallingford, UK

Mimee, Z., Dauphinais, N., and Bélair, G. 2015. Life cycle of the golden cys nematode, Globodera rostochiensis, in Quebec, Canada. J. Nematol. 47: 290-295.

Moxnes, J. F., and Hausken, K. 2007. The population dynamics of potato cyst nematodes. Ecol. Modell. 207:339-348.

Mugniery, D. 1978. Post embryonic development, in relation with temperature, of Globodera rostochiensis and G. pallida (Nematoda: Heteroderidae). Rev. Nematol. 1:3-12.

Perry, R. N. 1989. Dormancy and hatching of nematode eggs. Parasitol. Today 5:377-383.

Phillips, W. S., Kieran, S. R., and Zasada, I. A. 2015. The relationship between temperature and development in Globodera ellingtonae. J. Nematol. 47: 283-289.

Renčo, M. 2007. Comparison of the life cycle of potato cyst nematode (Globodera rostochiensis) pathotype Rol on selected potato cultivars. Biologia (Bratisl.) 62:195-200.

Ryan, A., and Devine, K. J. 2005. Comparison of the in-soil hatching responses of Globodera rostochiensis and G. pallida in the presence and absence of the host potato crop cv. British Queen. Nematology 7:587-597.

Scholte, K., and Vos, J. 2000. Effects of potential trap crops and planting date on soil infestation with potato cyst nematodes and root-knot nematodes. Ann. Appl. Biol. 137:153-164.

Skantar, A. M., Handoo, Z. A., Zasada, I. A., Ingham, R. E., Carta, L. K., and Chitwood, D. J. 2011. Morphological and molecular characterization of Globodera populations from Oregon and Idaho. Phytopathology 101: 480-491.

Turner, S. J. 1996. Population decline of potato cyst nematodes (Globodera rostochiensis, G. pallida) in field soils in Northern Ireland. Ann. Appl. Biol. 129:315-322.

Turner, S. J., and Evans, K. 1995. The origins, global distribution and biology of potato cyst nematodes (Globodera rostochiensis (Woll.) and G. pallida (Stone). Pages 7-26 in: Potato Cyst Nematodes: Biology, Distribution, and Control. R. J. Marks and B. B. Brodie, eds. CAB International, New York.

Zasada, I. A., Ingham, R. E., and Phillips, W. S. 2015. Biological insights into Globodera ellingtonae. Asp. Appl. Biol. 130:1-8.

Zasada, I. A., Peetz, A., Wade, N., Navarre, R. A., and Ingham, R. E. 2013. Host status of different potato (Solanum tuberosum) varieties and hatching in root diffusates of Globodera ellingtonae. J. Nematol. 45:195-201. 